An official journal of the / Un journal officiel de la "Société Sénégalaise de Cancérologie" (SOSECAN)

Journal homepage: www.africanjournalofoncology.com

Original article / Article original

DOI: https://www.doi.org/I0.54266/ajo.I.2.55.WMWE85I5

\title{
Profile of parotid cancers at the ENT clinic Lamine Sine Diop of Fann teaching hospital
}

\section{Profil des cancers de la parotide à la clinique ORL Lamine Sine Diop du CHU de Fann}

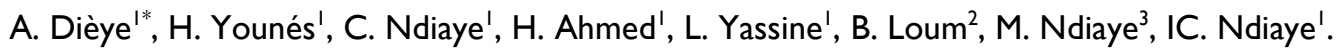

I Clinique ORL Lamine Sine Diop, CHU de Fann, Sénégal.

2 Service d'ORL, Hôpital d'Enfants Albert Royer, Sénégal.

${ }^{3}$ Service d'ORL, Hôpital pour enfants de Diamniadio, Sénégal.

INTRODUCTION: Parotid cancers are characterized by a great histological diversity and they pose diagnostic, therapeutic and evolutionary problems. We deliver through this study our experience on the management of malignant parotid tumors. MATERIALS AND METHODS: This is a retrospective, descriptive and analytical study carried out over a period of 12 years in the ENT department of Fann teaching hospital. RESULTS: The mean age at diagnosis was 48 years with a sex-ratio of I.6. The time to symptom progression was approximately 47 months. Parotid swelling was present in all patients and peripheral facial palsy was found in $31 \%$ of patients. Ultrasound of the parotid region was performed in II patients, i.e. $34 \%$, and computed tomography in 18 patients, i.e. $56 \%$. Fine needle aspiration was performed in II patients. Parotid cancers accounted for $44 \%$ of all parotid tumors. Sixty-eight percent of patients consulted at the T4 stage. Seventy-one percent of patients received surgical treatment. The most common histologic type was muco-epidermoid carcinoma. Five patients received radiotherapy. The postoperative effects were dominated by PFP (18\%). Seven cases of death were recorded. CONCLUSION: The management of parotid cancer still poses diagnostic and above all therapeutic problems. The combination of radiotherapy surgery, very rarely encountered in our study, provides a better prognosis and better survival.

KEYWORDS: Parotid; Cancer; Parotidectomies; Radiotherapy.

INTRODUCTION : Les cancers de la parotide sont caractérisés par une grande diversité histologique et ils posent des problèmes diagnostiques, thérapeutiques et évolutifs. Nous livrons à travers cette étude notre expérience sur la prise en charge des tumeurs malignes de la parotide. MATERIELS ET METHODES : Il s'agit d'une étude rétrospective, descriptive et analytique réalisée sur une période de 12 ans dans le service d'ORL du CHNU de Fann. RESULTATS : L'âge moyen au moment du diagnostic était de 48 ans avec un sex-ratio de I,6. La durée d'évolution des symptômes était d'environ de 47 mois. La tuméfaction parotidienne était présente chez tous les patients et une paralysie faciale périphérique était retrouvée chez $31 \%$ des patients. L'échographie de la région parotidienne a été réalisée chez II patients, soit 34\% et la tomodensitométrie chez 18 patients, soit $56 \%$. La cytoponction était effectuée chez II patients. Les cancers parotidiens représentaient $44 \%$ de l'ensemble des tumeurs parotidiennes. Soixantehuit pour cent des patients ont consulté au stade de T4. Soixante-onze pourcent des patients ont bénéficié d'un traitement chirurgical. Le type histologique le plus fréquent était le carcinome muco-épidermoïde. Cinq patients ont bénéficié de radiothérapie. Les suites opératoires ont été dominées par la PFP (18\%). Sept cas de décès ont été enregistrés. CONCLUSION : Le profil des cancers de la parotide est caractérisé par l'âge jeune, le stade avancé au diagnostic et l'absence de traitement adapté caractérisé en dehors de la chirurgie par la rareté de la radiothérapie et de la chimiothérapie.

MOTS-CLES : Parotide ; Cancer ; Parotidectomies ; Radiothérapie.

\section{INTRODUCTION}

Les cancers de la parotide représentent $20 \%$ des tumeurs parotidiennes [ 1,2$]$. Les cancers parotidiens sont caractérisés par leur grande diversité de présentation clinique, histologique, d'évolution et de pronostic [3]. Le type histologique le plus fréquent est le carcinome muco-épidermoïde.

Le but de notre travail rétrospectif était d'étudier les aspects épidémiologiques, la présentation clinique des cancers parotidiens, leurs aspects histologiques thérapeutiques et évolutifs et de définir les facteurs pronostiques au sein de la clinique d'ORL et de chirurgie cervico-faciale du CHU de Fann.

\section{MATERIELS ET METHODES}

II s'agit d'une étude rétrospective, descriptive et analytique réalisée sur une période de 10 ans (premier Octobre 2009 au 3I Décembre 2019) dans le service d'ORL du CHNU de Fann. Nous avons inclus dans notre étude tout patient ayant consulté pour cancer de la parotide opéré et non opéré histologiquement documenté. Les paramètres étudiés étaient épidémiologiques, cliniques, paracliniques, histologiques, thérapeutiques et évolutifs. L'analyse des données a été faite par le logiciel Microsoft Excel@C 2010.

\section{RESULTATS}

Sur un total de 63 patients présentant une tumeur de la parotide, 32 souffraient de cancers soit $50 \%$. 
Données sociodémographiques

L'âge moyen était de 48 ans avec des extrêmes allant de II à 89 ans avec un pic entre 40 et 60 ans (Figure I).

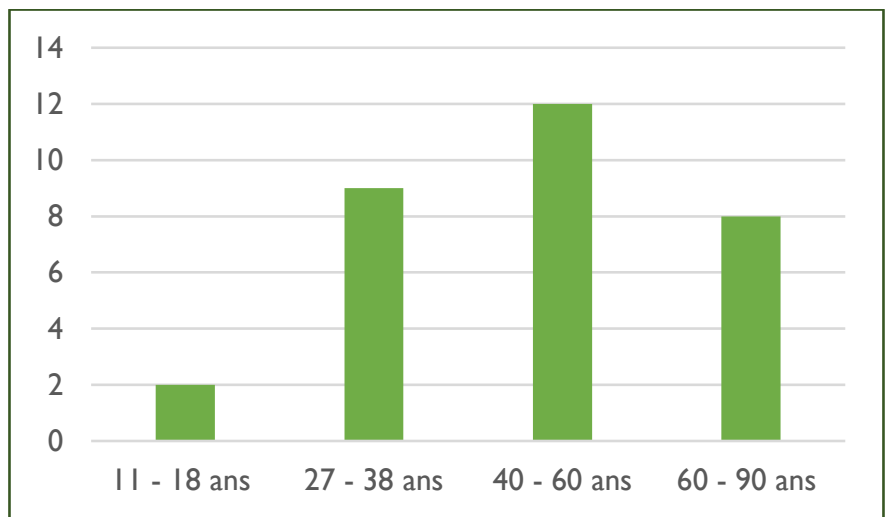

Figure I : Répartition selon l'âge.

Le sex-ratio était de I,6 avec une prédominance masculine (20 hommes sur 12 femmes). Le délai médian de consultation était de 47 mois avec des extrêmes allant de 4 mois à 30 ans.

\section{Données cliniques}

Tous nos patients ont présenté une tuméfaction de la région parotidienne au moment de la consultation (Figure 2).

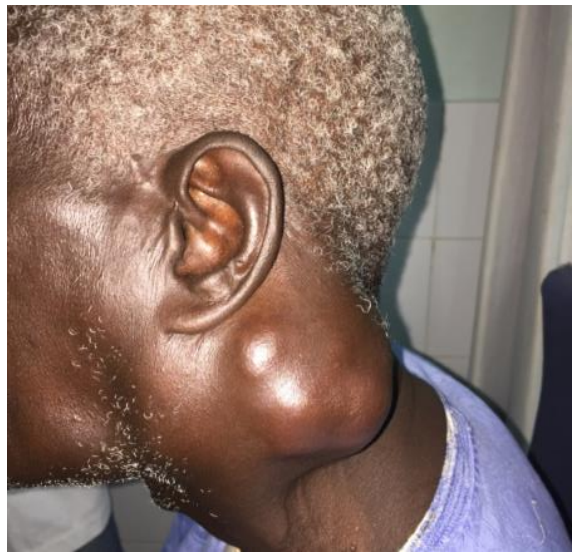

Figure 2 : Tuméfaction parotidienne rétro-angulomaxillaire gauche.

La taille moyenne de la masse parotidienne était de huit $\mathrm{cm}$ avec des extrêmes de trois à $20 \mathrm{~cm}$. Le siège de la tuméfaction était sous lobulaire dans 17 cas (53\%). Elle était dure dans 19 cas $(59 \%)$, ferme dans II cas $(34 \%)$ et fixée dans six cas. Elle était associée à des douleurs locales dans neuf cas (28\%), à une paralysie faciale périphérique dans 10 cas (31\%), à des adénopathies suspectes dans huit cas (25\%). Une atteinte cutanée était retrouvée dans 14 cas (43\%) faite de lésion ulcéro-bourgeonnante ou de fistulisation. Nous notions également un trismus dans deux cas (Figure 3).

\section{Données paracliniques}

L'échographie a été effectuée dans II cas, soit 34\%, montrant dans tous les cas une formation tissulaire hétérogène suspecte. La tomodensitométrie (TDM) réalisée dans 18 cas, soit $56 \%$, montrait une tumeur isolée de la parotide dans trois cas, une tumeur avec extension locorégionale dans 15 cas, soit $46 \%$ et des métastases pulmonaires dans quatre cas (Figure 4).

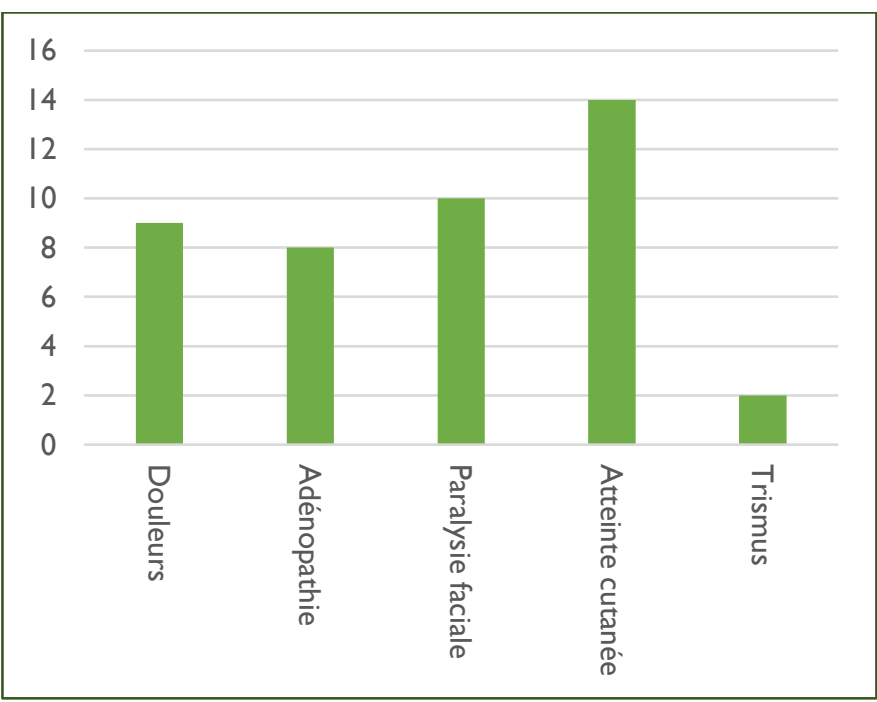

Figure 3 : Signes cliniques associés.

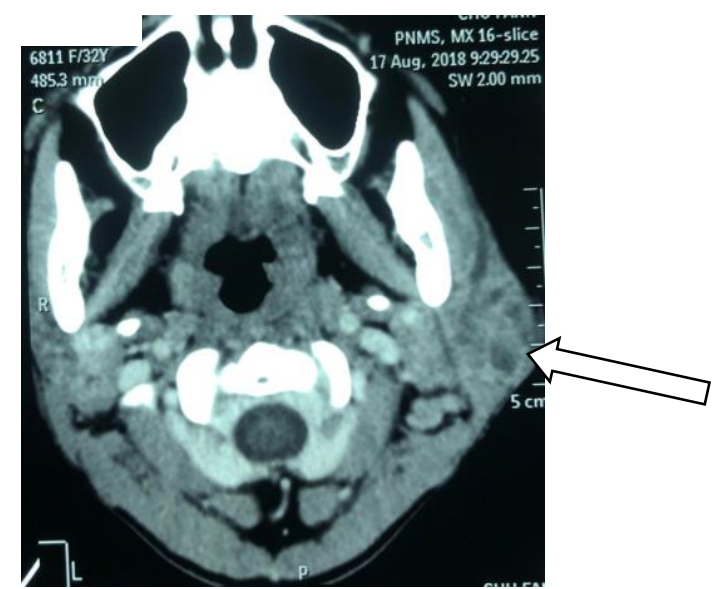

Figure 4: TDM en coupe axiale correspondant à un carcinome muco-épidermoïde de la parotide droite (flèche blanche) [Source : Collection ORL Fann]

L'imagerie par résonance magnétique (IRM) a été faite dans deux cas, soit $8 \%$ et elle objectivait une lésion étendue au lobe temporal gauche ou à la fosse infra-temporale. La cytoponction a été pratiquée dans II cas, soit $34 \%$. Elle montrait une lésion suspecte de malignité dans trois cas, soit $9 \%$, une lésion maligne dans un cas, une lésion bénigne dans six cas, soit $18 \%$, elle était non contributive dans un cas. Son association avec l'IRM a été effectuée dans un cas et la cytoponction montrait une lésion bénigne avec des atypies modérées, il s'agissait en effet d'un cylindrôme. Soixante-huit pour cent des patients ont consulté au stade de T4 (2I cas), dans quatre cas, la lésion était classée T2, dans trois cas, elle était classée T3. Quatre patients ont présenté des métastases.

\section{Données thérapeutiques}

Sur le plan chirurgical, 10 patients, soit $27 \%$ ont bénéficié d'une parotidectomie totale non conservatrice (PTNC) et neuf patients, soit $28 \%$ ont bénéficié d'une parotidectomie totale conservatrice (PTC), quatre patients ont bénéficié d'une parotidectomie totale élargie (PTE). Un curage ganglionnaire a été réalisé dans neuf cas, soit $28 \%$. II s'agissait d'un curage fonctionnel dans cinq cas, d'un curage radical dans deux cas et d'un curage triangulaire dans deux cas. La reconstruction des pertes de substances cutanées a été faite à l'aide de lambeaux 
pédiculés myo-cutané du grand pectoral ou temporo-frontal dans quatre cas (Figure 5).

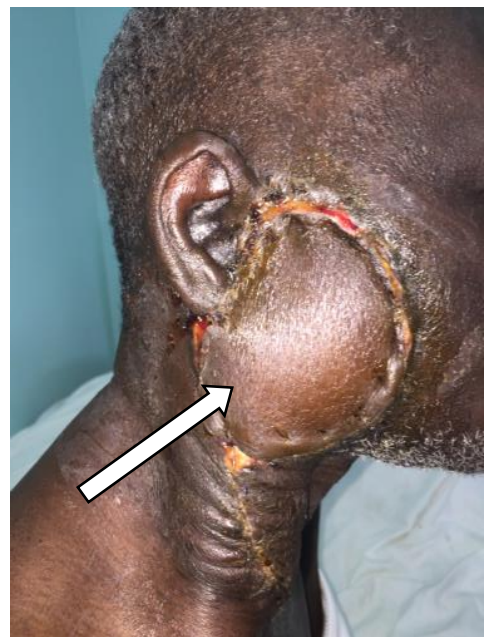

Figure 5 : LMCGP (flèche blanche) après PTNC droite pour carcinome mucoépidermoïde de haut grade étendu au CAE.

La radiothérapie a été effectuée dans six cas, soit $18 \%$. Sept patients ont bénéficié d'une chimiothérapie, parmi eux, quatre présentaient des lésions inopérables.

\section{Données pathologiques}

L'analyse anatomo-pathologique des pièces opératoires ou des biopsies avait révélé une grande diversité histo-pathologique (Tableau I).

Tableau I : Répartition histologique.

\begin{tabular}{|lll|}
\hline Type histologique & Effectif & Pourcentage (\%) \\
\hline $\begin{array}{l}\text { Carcinome muco- } \\
\text { épidermoïde }\end{array}$ & 9 & 28 \\
$\begin{array}{l}\text { Carcinome } \\
\text { épidermoïde }\end{array}$ & 6 & 18 \\
$\begin{array}{l}\text { Carcinome adénoïde } \\
\text { kystique }\end{array}$ & 5 & 15 \\
\begin{tabular}{l} 
Adénocarcinome \\
\hline
\end{tabular} & 4 & 12 \\
\hline
\end{tabular}

\section{Evolution}

Les suites précoces étaient une paralysie faciale périphérique dans six cas, une infection du site opératoire (ISO) dans quatre cas, une nécrose et une infection du lambeau dans trois cas, une parésie dans trois cas, un hématome, ISO et une nécrose dans un cas et une nécrose du site opératoire dans un cas. Les suites tardives étaient un syndrome de Frey dans deux cas, une poursuite évolutive dans quatre cas (à un mois, quatre mois, sept mois et huit mois) et une radiodermite dans un cas. Quatorze patients, soit $43 \%$ ont été perdus de vue et sept cas de décès ont été déplorés. Les suites ont été simples pour six patients. Le recul moyen était de 20 mois.

\section{DISCUSSION}

Les cancers de la parotide représentent 20 à $40 \%$ des tumeurs de la parotide, ce chiffre est légèrement inférieur à celui retrouvé dans notre série qui est de $50 \%$. L'âge moyen constaté de 48 ans est retrouvé par plusieurs auteurs $[4,5]$. La prédominance masculine notée dans plusieurs travaux est également caractéristique des cancers de la parotide $[6,7]$. Les longs délais de consultation dans notre contexte sont liés aux difficultés d'accès aux soins ORL dans certaines localités, à la négligence mais aussi à la peur de la chirurgie. Ces longs délais font découvrir des formes géantes de tumeur parotidienne, mais aussi des lésions très évoluées non éligibles à une chirurgie carcinologique [4]. La malignité des tumeurs parotidiennes est suspectée en présence de douleur, de paralysie faciale, d'atteinte ganglionnaire, d'atteinte cutanée ou de trismus [4, 8-II]. Pour certains auteurs, la douleur est associée aux tumeurs malignes dans $25 \%$, ce qui corrobore nos résultats [12]. La présence de parésie ou de paralysie faciale est estimée entre 12 et $20 \%$ [10]. Ce chiffre est inférieur à celui retrouvé dans notre étude donnant à l'atteinte nerveuse un véritable facteur prédictif de cancer. Le risque de PFP dépend de certains facteurs à savoir l'âge avancé, le type et le grade histologiques, le délai de chirurgie, l'extension tumorale, une chirurgie secondaire ou de récidive [13]. L'atteinte ganglionnaire évaluée entre 10 à $20 \%$ est également reconnue comme étant un facteur de récidive et de métastases surtout pulmonaires.

Sur le plan radiologique, l'échographie est l'examen de base, non invasif, de réalisation simple et rapide [14]. Certains signes échographiques tels que le caractère hétérogène, mal limité et à contours irréguliers de la tumeur ainsi qu'une vascularisation intense peut être suspects de malignité [14]. La TDM est devenue un examen de plus en plus utilisée dans nos conditions d'exercice [56\%], contrairement il y a une quinzaine d'années où elle était de $13 \%$ [4]. Elle permet de préciser la topographie des lésions et leurs extensions aux structures adjacentes surtout osseuses. L'IRM réalisée dans trois cas, soit $8 \%$ pose toujours un problème d'accessibilité dans notre pratique du fait de son cout élevé et de sa disponibilité. L'IRM apparait actuellement comme l'examen préférentiel dans l'exploration de la pathologie tumorale de la glande parotide [16]. L'infiltration des espaces péri-glandulaires fait évoquer une tumeur maligne, de même que la présence d'adénopathies nécrotiques et d'une capsule glandulaire mal limitée et irrégulière, elle permet aussi la discrimination bénigne-maligne dans $80 \%$ des cas, possible grâce au coefficient d'ADC (coefficient apparent de diffusion) dont des valeurs basses sont en faveur d'une lésion maligne [16, 17]. Cette différenciation est de $92 \%$ lorsqu'elle est couplée à la cytoponction échoguidée [17].

Dans notre contexte, la cytoponction ne permet pas d'éviter la chirurgie radicale mais permet de planifier le traitement des aires ganglionnaires, mais aussi de donner une meilleure information au patient. Le taux de faux négatif est de $31 \%$ dans la littérature, du fait de la grande diversité histologique des tumeurs parotidiennes rendant l'examen cytologique difficile [18]. Longuet et al [18] retrouvait une sensibilité et une spécificité de $81 \%$ et $97,5 \%$ respectivement, taux similaire avec ceux de Lim et al [19] avec $80 \%$ de sensibilité et de $100 \%$ de spécificité. Son association avec l'IRM permettent de planifier le geste opératoire (parotidectomie exofaciale ou totale et évidement cervical), mais ne guident pas l'attitude sur le nerf facial [9]. Cette association a été effectuée dans un seul cas dans notre étude et il s'agissait d'un cylindrôme. Le manque de moyens, l'inaccessibilité à l'IRM et les faux négatifs de la cytoponction font que cette association tarde à être régulièrement utilisée couramment dans nos structures. 
Les tumeurs malignes représentent $20 \%$ des tumeurs parotidiennes [6], nos résultats montraient $50 \%$ de tumeurs malignes. Le carcinome muco-épidermoïde est habituellement le plus fréquent [20] (Tableau II). Erovic et al [5] avait retrouvé un taux de $32 \%$, chiffre similaire à celui retrouvé dans notre étude avec $28 \%$.

\begin{tabular}{|c|c|}
\hline Auteurs & Types histologiques \\
\hline $\begin{array}{l}\text { Lierop et al } \\
\text { (Afrique du Sud, } \\
\text { 2007) [3] }\end{array}$ & $\begin{array}{l}\text { Carcinome épidermoide } \\
\text { Carcinome muco-épidermoide } \\
\text { Mélanome }\end{array}$ \\
\hline $\begin{array}{l}\text { Lee et al (Brésil, } \\
2018 \text { ) [27] }\end{array}$ & $\begin{array}{l}\text { Carcinome canalaire } \\
\text { Carcinome muco-épidermoide } \\
\text { Carcinome épidermoide }\end{array}$ \\
\hline $\begin{array}{l}\text { Paris et al } \\
\text { (France, 2004) } \\
\text { [22] }\end{array}$ & $\begin{array}{l}\text { Carcinome muco-épidermoide } \\
\text { Carcinome adénoïde kystique } \\
\text { Carcinome ex adénome pléomorphe }\end{array}$ \\
\hline $\begin{array}{l}\text { Beltaief (Tunisie, } \\
\text { 2007) [8] }\end{array}$ & $\begin{array}{l}\text { Carcinome sur tumeur mixte } \\
\text { Carcinome muco-épidermoide } \\
\text { Maladie de Hodgkin }\end{array}$ \\
\hline $\begin{array}{l}\text { Venkatesh (Inde, } \\
\text { 2019) [2I] }\end{array}$ & $\begin{array}{l}\text { Carcinome muco-épidermoide } \\
\text { Carcinome ex adénome pléomorphe } \\
\text { Adénocarcinome }\end{array}$ \\
\hline $\begin{array}{l}\text { Notre étude } \\
\text { (Sénégal, 2020) }\end{array}$ & $\begin{array}{l}\text { Carcinome muco-épidermoide } \\
\text { Carcinome épidermoide } \\
\text { CAK / Adénocarcinome }\end{array}$ \\
\hline
\end{tabular}

Les lésions classées T3-T4 prédominent dans nos études comme l'illustre ce travail où $64 \%$ des patients ont consulté au stade de T4 et trois patients au stade de T3. Ces résultats s'opposent à ceux de Paris et al [22] où $74 \%$ des patients étaient classés TI-T2 avec $86 \%$ de patients classés N0. Ces formes avancées rencontrées dans notre étude expliquent le recours aux lambeaux de reconstruction et la fréquence des poursuites évolutives responsables d'une lourde mortalité.

Devant toute tumeur maligne de la parotide, la parotidectomie totale est la règle associée ou non à un curage ganglionnaire et/ou à une radiothérapie [6]. Et l'enjeu chirurgical sera une chirurgie carcinologique avec de bonnes marges de sécurité et la préservation du nerf facial dans la mesure du possible. La PTNC est systématique dans notre pratique, devant toute PFP en préopératoire, de même que lorsqu'il existe une extension au nerf facial découverte en peropératoire. Cette attitude maximaliste est liée à l'absence d'examen extemporané et de monitoring du nerf facial, mais aussi à la difficulté de payer une seconde intervention [4, 23]. Le curage est systématique en cas d'adénopathies palpables pour les tumeurs T2-T4 de haut grade de malignité, pour les TI, un curage sélectif est fait et sera complété si positif à l'examen extemporané par un curage fonctionnel [24]. L'évidemment ganglionnaire est discuté pour les tumeurs de bas grade [II]. L'examen extemporané peut guider l'attitude sur les ganglions en fonction de la fréquence des adénopathies selon le type histologique. La difficulté d'accès à l'examen extemporané au Sénégal ne permet pas de réaliser un curage sélectif devant un cas avéré de cancer parotidien, justifiant l'attitude maximaliste à réaliser une PTC avec curage fonctionnel devant tout cas de tumeur suspecte de malignité de la glande parotide. La majorité des adénopathies
(66\%) dans cette présente étude étaient retrouvées dans les carcinomes épidermoïdes, dans $22 \%$, il s'agissait d'adénocarcinome et de carcinome muco-épidermoïde dans $22 \%$. La discussion d'une parotidectomie ne se fait pas sans parler du nerf facial. La parotidectomie peut être élargie au nerf facial en cas d'atteinte de celui-ci. L'attitude vis-à-vis du nerf facial n'est pas codifiée. Certains auteurs craignant de léser le nerf facial, le conservent même s'il est macroscopiquement envahi et complètent par une radiothérapie [9]. Cependant l'objectif d'une chirurgie pour cancer de la parotide est d'être carcinologique [10]. Dans notre série, ce sacrifice a été réalisé dans 10 cas, soit $27 \%$, illustrant l'importance des lésions étendues au nerf facial. Concernant le traitement complémentaire, plusieurs études confirment l'amélioration du pronostic et du taux de survie en cas d'association chirurgieradiothérapie par augmentation du taux de contrôle locorégional de 57 à $83 \%$ en cas de $\mathrm{NI}[5,8,9,25,26]$. Pour les patients N0, le traitement des aires ganglionnaires fait toujours l'objet de débat. Les facteurs prédictifs d'extension ganglionnaire empiriques restent le type histologique, le grade histologique, la taille de la tumeur [9]. Dans notre étude, plusieurs protocoles de fin d'irradiation n'ont pas été retrouvés rendant difficile la comparaison avec d'autres études. Les indications actuellement admises par la plupart des auteurs $[3,9,17]$ sont des limites d'exérèse chirurgicales envahies, une invasion lymphatique, vasculaire et péri-neurale, des tumeurs de haut grade de malignité, une extension tumorale extraglandulaire, des adénopathies envahies et une extension au lobe profond.

Concernant les complications, la PFP était objectivée dans $24 \%$ des cas. Un taux similaire (30\%) était retrouvé dans l'étude de Lee et al [27], ce chiffre est proche de l'incidence globale de la parésie dans la littérature [28]. Le syndrome de Frey a été retrouvé dans $5 \%$ dans cette série. Le taux de récidive était faible (un patient). Il était de $22 \%$ dans l'étude de Erovic et al [5]. Cette différence peut s'expliquer par le faible taux de radiothérapie post-opératoire (18\%). Les facteurs pronostiques péjoratifs évoqués par plusieurs auteurs sont la taille de la lésion (supérieure à quatre $\mathrm{cm}$ ), la présence d'adénopathies cervicales, l'atteinte du nerf facial, le type histologique, le grade histologique élevé, des marges d'exérèse insuffisantes, une invasion lymphatique et péri neurale et l'âge supérieur à 55 ans [3, 5]. La survie est de 70 à $100 \%$ à cinq ans pour les carcinomes muco-épidermoïdes de bas grade et de zéro à $50 \%$ à cinq ans pour les carcinomes muco-épidermoïdes de haut grade, les cylindrômes et les épidermoïdes [7]. Dans cette série, $40 \%$ des carcinomes muco-épidermoïdes étaient de haut grade.

\section{CONCLUSION}

Les cancers de la parotide sont relativement rares, ils sont caractérisés par une grande diversité histologique et ils posent des problèmes diagnostiques, thérapeutiques et évolutifs. L'imagerie est d'un apport indéniable dans le diagnostic. La cytoponction peu réalisée dans notre étude, constitue un élément utile dans l'orientation diagnostique et la décision thérapeutique lorsqu'elle est couplée à l'IRM. Les longs délais de consultation expliquent la fréquence des stades avancés et l'attitude maximaliste dans notre pratique. Le développement de l'examen extemporané permettrait de limiter les gestes excessifs. La chirurgie carcinologique suivie d'une radiothérapie permet d'assurer un meilleur contrôle 
locorégional de la tumeur et une survie meilleure. Ce traitement n'est pas toujours disponible chez nos patients.

\section{CONFLITS D'INTERET}

Les auteurs n'ont déclaré aucun conflit d'intérêts.

\section{REFERENCES}

I. Diom E-S, Thiam A, Tall A, Ndiaye M, Toure S, Diouf R. Profil des tumeurs de la glande parotide: expérience sur 93 cas colligés en I6ans. Ann Fr OtoRhino-Laryngol Pathol Cervico-Faciale. févr 2015; I32(1):9-12.

2. Golar R, C. Anatomie chirurgicale de la région parotidienne. 95(6):395 - 4I0.

3. Lierop ACV, Fagan JJ. Parotidectomy in Cape Town a review of pathology and management. SAJS. 2007. 45;3. 96-103.

4. Diom Es, Déguenonvo Rea, Tall A, Ndiaye M, Diop A. Aspects chirurgicaux des cancers parotidiens au SénégaL: A propos de 30 cas. RevCAMES-Sér A. 20I2; I3((I)):63-5.

5. Erovic BM, Shah MD, Bruch G, Johnston M, Kim J, O'Sullivan B, et al. Outcome analysis of 215 patients with parotid gland tumors: a retrospective cohort analysis. J Otolaryngol - Head Neck Surg. déc 2015;44(I):43.

6. Bouaity B, Darouassi* $Y$, Chihani M, Touati MM, Ammar $\mathrm{H}$. Les facteurs prédictifs de malignité dans la prise en charge des tumeurs parotidiennes: à propos de 76 cas. Pan Afr Med J 2016. 23; II 2.

7. Vincentiis MD, Magliulo G, Soldo P, Manciocco V, Pagliuca G, Gaizo RD et al. Extended parotidectomy. Acta Otorhinolaryngol Ital 2005. 25, 169-I73,

8. Beltaief N, Tababi S, Atallah S, Mansour M, Ouertatani I, Charfi A, et al. Les Tumeurs Malignes De La Parotide. J Tunis ORL Chir Cervico-Faciale. 25 août 2008; | (1):25-8.

9. Bouyon A, Hans S, Durdux C, Housset M. Tumeurs malignes de la parotide: prise en charge multidisciplinaire, rôle de la radiothérapie. Cancer/Radiothérapie. déc 2007; I I (8):465-75.

10. Cracchiolo JR, Shaha AR. Parotidectomy for Parotid Cancer. Otolaryngol Clin North Am. avr 2016;49(2):4I5-24.

I I. Spiro RH. Management of malignant tumours of the salivary glands. Oncology. 1998;(I2):67I-83.

12. Prades J-M, Oletski A, Faye M-B, Dumollard J-M, Timoshenko A-P, Veyret $C$, et al. Morphologie IRM des tuméfactions de la glande parotide. Corrélations histopathologiques. Morphologie. mars 2007;9I (292):44-5 I.

13. Ikoma R, Ishitoya J, Sakuma Y, Hirama M, Shiono O, Komatsu $M$, et al. Temporary facial nerve dysfunction after parotidectomy correlates with tumor location. Auris Nasus Larynx. oct 20I4;4I(5):479-84.

14. Bruneton J, Mourou M. Ultrasound in salivary gland disease. ORL. 1993;(55):284-9.

15. Makeieff M, Guerrier B, Prades J-M, Reyt E, Zanaret M. Pathologie maligne de la parotide. Ann Otolaryngol Chir Cervico-Faciale. avr 2009; I 26(2):75-8I.
16. Raine C, Saliba K, Chippindale A J, et al. Radiological imaging in primary parotid malignancy. British Journal of Plastic Surgery 2003; 56:637-643.

17. Maruo T, Fujimoto Y, Yoshida K, Hiramatsu M, Suzuki A, Nishio $\mathrm{N}$, et al. Effect of clinical symptoms on the indication for selective neck dissection for NO carcinomas of the parotid gland. Oncol Lett. juill 2014;8(I):335-8.

18. Longuet M, Nallet E, Guedon C, Depondt J. Valeur diagnostique de la cytoponction et de l'examen histologique extemporané dans les tumeurs parotidiennes primitives opérées. Rev Laryngol Otol Rhinol. 200I; I (I22):5 I-5.

19. Lim CM, They J, Loh KS, Chao SS, Lim LHY, Tan LKS. Role of fine-needle aspiration cytology in the evaluation of parotid tumours. ANZ J Surg. sept 2007;77(9):742-4.

20. Sood S, McGurk M, Vaz F. Management of Salivary Gland Tumours: United Kingdom National Multidisciplinary Guidelines. J Laryngol Otol. mai 2016;130(S2):SI42-9.

21. Venkatesh S, Srinivas T, Hariprasad S. Parotid gland tumors: 2-year prospective clinicopathological study. Ann Maxillofac Surg. 2019;9(I):103.

22. Paris J, Coulet O, Facon F, Chrestian M-A, Giovanni A, Zanaret $M$. Cancers primitifs de la parotide: approche anatomo-clinique. Rev Stomatol Chir Maxillofac. déc 2004; I05(6):309-I5.

23. Diop E, Hitimana A, Diouf R, Diop LS. Bilan de 40 parotidectomies. Rev Laryngol Otol Rhinol. 1984;(105):25-31.

24. Harish K. Management of primary malignant epithelial parotid tumors. Surg Oncol. juill 2004;13(1):7-16.

25. Kaur J, Goyal S, Muzumder S, Bhasker S, Mohanti BK, Rath GK. Outcome of Surgery and Post-Operative Radiotherapy for Major Salivary Gland Carcinoma: Ten Year Experience from a Single Institute. Asian Pac J Cancer Prev. 23 oct 2014; I5(19):8259-63.

26. Terhaard $\mathrm{CHJ}$, Lubsen $\mathrm{H}$, Rasch $\mathrm{CRN}$, Levendag PC, Kaanders HHÀM, Tjho-Heslinga RE, et al. The role of radiotherapy in the treatment of malignant salivary gland tumors. Int J Radiat Oncol. janv 2005;6I(I):I03-II.

27. Lee DH, Yoon TM, Lee JK, Lim SC. Clinicopathologic factors associated with recurrence in parotid carcinoma. Braz J Otorhinolaryngol. nov 20I8;84(6):69I-6.

28. Jin H, Kim BY, Kim H, Lee E, Park W, Choi S, et al. Incidence of postoperative facial weakness in parotid tumor surgery: a tumor subsite analysis of 794 parotidectomies. BMC Surg. déc 2019;19(I):199. 